\title{
CrossPlan: Systematic Planning of Genetic Crosses to Validate Mathematical Models
}

\author{
Aditya Pratapa \\ Dept. of Computer Science, \\ Virginia Tech, Blacksburg, VA \\ Nicholas Franzese \\ Department of Biology, Reed College \\ Portland, OR
}

\author{
Neil Adames \\ Dept. of Chemical \& Biological \\ Engineering, Colorado State \\ University, Fort Collins, CO \\ John Tyson \\ Dept. of Biological Sciences, \\ Virginia Tech, Blacksburg, VA
}

\author{
Pavel Kraikivski \\ Dept. of Biological Sciences, \\ Virginia Tech, Blacksburg, VA \\ Jean Peccoud \\ Dept. of Chemical \& Biological \\ Engineering, Colorado State \\ University, Fort Collins, CO
}

\author{
T. M. Murali* \\ Dept. of Computer Science, Virginia \\ Tech, Blacksburg, VA
}

\begin{abstract}
Mathematical models of cellular processes can systematically predict the phenotypes of novel combinations of multi-gene mutations. Searching for informative predictions and prioritizing them for experimental validation is challenging since the number of possible combinations grows exponentially in the number of mutations. Moreover, keeping track of the crosses needed to make new mutants and planning sequences of experiments is unmanageable when the experimenter is deluged by hundreds of potentially informative predictions to test.

We present CrossPlan, a novel methodology for systematically planning genetic crosses to make a set of target mutants from a set of source mutants [1]. We base our approach on a generic experimental workflow used in performing genetic crosses in budding yeast. We prove that the CROSSPLAN problem is NP-complete. We develop an integer-linear-program (ILP) to maximize the number of target mutants that we can make under certain experimental constraints. We apply our method to a comprehensive mathematical model of the protein regulatory network controlling cell division in budding yeast. We also extend our solution to incorporate other experimental conditions such as a delay factor that decides the availability of a mutant and genetic markers to confirm gene deletions. The experimental flow that underlies our work is quite generic and our ILP-based algorithm is easy to modify. Hence our framework should be relevant in plant and animal systems as well.

This paper opens up a new area of research: how to automatically synthesize efficient experimental plans for making large numbers of mutants carrying perturbations in multiple genes. Moreover, the
\end{abstract}

\footnotetext{
${ }^{*}$ Corresponding author. Contact: murali@cs.vt.edu

Permission to make digital or hard copies of part or all of this work for personal or classroom use is granted without fee provided that copies are not made or distributed for profit or commercial advantage and that copies bear this notice and the full citation on the first page. Copyrights for third-party components of this work must be honored

For all other uses, contact the owner/author(s).

ACM-BCB'18, August 29-September 1, 2018, Washington, DC, USA

(C) 2018 Copyright held by the owner/author(s)

ACM ISBN 978-1-4503-5794-4/18/08.

https://doi.org/10.1145/3233547.3233718
}

principles used in CROSSPLAN can be directly extended to other organisms where siRNA or CRISPR-based screens are effective. Thus the growing community of biomedical scientists who are beginning to use CRISPR-based approaches to plan multiple, combinatorial gene perturbations will find our approach to be very relevant to their research.

\section{KEYWORDS}

experiment planning, genetic crosses, multi-gene mutants, integer linear program, mathematical model simulations

\section{ACM Reference Format:}

Aditya Pratapa, Neil Adames, Pavel Kraikivski, Nicholas Franzese, John Tyson, Jean Peccoud, and T. M. Murali. 2018. CrossPlan: Systematic Planning of Genetic Crosses to Validate Mathematical Models. In ACM-BCB'18: 9th ACM International Conference on Bioinformatics, Computational Biology and Health Informatics, August 29-September 1, 2018, Washington, DC, USA. ACM, Washington DC, USA, 1 page. https://doi.org/10.1145/3233547.3233718

\section{REFERENCES}

[1] Aditya Pratapa, Neil Adames, Pavel Kraikivski, Nicholas Franzese, John J. Tyson, Jean Peccoud, and T. M. Murali. 2018. CrossPlan: Systematic Planning of Genetic Crosses to Validate Mathematical Models. Bioinformatics 34, 13 (2018), 2237-2244. 